\title{
Probable trigeminal nerve schwannoma in a dog
}

J.H. Saunders ${ }^{+}$; DVM, L. Poncelet, DVM, PhD, C. Clercx ${ }^{+}$, DVM, PhD, F.R. Snaps ${ }^{+}$, DVM, P. Flandroy ${ }^{++}$, MD, P. Capasso ${ }^{+++}$, MD, R.F. Dondelinger ${ }^{++}$, MD.

+ From the Departments of Medical Imaging (Saunders, Snaps); Small Animal Internal Medecine (Clercx) and Small Animal Surgery (Poncelet) of the Faculty of Veterinary Medicine, University of Liège-B41, 4000 Liège, Belgium.

++ From the Department of Medical Imaging, University Hospital Center-B35, University of Liège, 4000 Liège, Belgium.

+++ From the Department of Diagnostic and Interventional Radiology; University Hospital Center - CHUV; 1011-Lausanne, Switzerland. 
Correspondence and reprint requests to Dr J.H. Saunders, Department of Medical Imaging, Faculty of Veterinary Medicine, University of Liège, Sart-Tilman - B41, 4000 Liège, Belgium.

A 7-year-old male husky dog developed atrophy of the right masseter muscle and pruritis of the right side of the face. A myogenic origin was excluded using muscular biopsy. Electrophysiologically, there was involvement of the motor and sensory fibers of the trigeminal nerve suggesting a lesion located between the brainstem and the trigeminal ganglion. On MRI examination, a nodular mass was detected in the right caudal fossa. This mass was characterized by intense enhancement after injection of contrast medium. Due to the progressive clinical signs, electrophysiology and MRI results, a presumptive diagnosis of a trigeminal nerve schwannoma was made. The animal's condition improved slightly with 
corticosteroids. The dog was euthanazied 3 months after initial presentation. Necropsy was not performed.

Key words: dog, MRI, trigeminal nerve, schwannoma, diagnostic imaging

\section{Case history}

A 7-year-old male husky dog, weighing $25 \mathrm{~kg}$, was presented to the Department of Small Animal Internal Medicine of our institution with a history of right muscular atrophy of the masticatory muscles and severe pruritis of the right side of the face. At that time, the dog was afebrile and in good general condition. Severe atrophy of the right masticatory muscles was present, most obvious at the level of the temporal muscle. The right eyelid was oedematous. The dog could normally open and close its mouth.

An electrophysiological examination was performed. The dog was sedated with medetomedine* (50 $\mu \mathrm{g} / \mathrm{kg}, \mathrm{IM})$ and general anesthesia was induced and 
maintained with sodium pentobarbital $\dagger(5 \mathrm{mg} / \mathrm{kg}, \mathrm{IV})$. Needle electromyography disclosed spontaneous activity in the right masticatory muscles. Facial nerve function was evaluated through stimulation of the main trunk. Facial nerve recording was performed with a subcutaneous needle at the level of the orbicularis oculi muscle referenced to an electrode of the same type inserted at the level of the medial angle of the eye. Then, with the recording electrodes in the same location, the maxillary branch of the trigeminal nerve was stimulated at its emergence from the maxillary foramen. ${ }^{1}$ The procedure was conducted on both sides of the head and the latencies were compared. The latencies in the auriculopalpebral branch of the facial nerves were the same on both sides. There was a twofold increase in latency of the right trigeminofacial reflex (16.2 msec on the right side compared to 8.4 msec for the reflex on the left side). During the same anesthesia, the right temporalis muscle was biopsied and placed in a $10 \%$ buffered solution for hematoxylin-eosin staining. Histopathologically, there was scattered muscle fiber atrophy without inflammatory reaction, most compatible with neurogenic atrophy. Magnetic resonance imaging was performed with a $1.5 \mathrm{~T}$ superconducting magnet. $\neq$ The dog was sedated with medetomedine* $(50 \mu \mathrm{g} / \mathrm{kg}, \mathrm{IM})$ and general anesthesia was induced and maintained with sodium pentobarbital $\dagger$ $\mathrm{mg} / \mathrm{kg}, \mathrm{IV})$. The patient was placed in sternal recumbency with the head in a knee 
coil. A two-dimensional Fourier transform (2DFT) fast low angle shot (FLASH) sequence was first obtained for localization in the transverse, dorsal and sagittal planes. Pre- and postcontrast T1-weighted spin-echo images were obtained with repetition times (TR) of $660 \mathrm{msec}$ and echo-times (TE) of $14 \mathrm{msec}$. T2-weighted spin-echo images were obtained with a TR of $6000 \mathrm{msec}$ and a TE of $90 \mathrm{msec}$. The slice-thickness was $3 \mathrm{~mm}$ and the images were overlapped to eliminate interslice gap. The matrix was 160 by 256 for both sequences. Image acquisition was performed in transverse, sagittal and dorsal planes before and after intravenous contrast medium administration (gadoterate meglumine $\$$, dose of $0.2 \mathrm{ml} / \mathrm{kg}$ ).

On the images, there was an $8 \mathrm{~mm}$ diameter, well-defined, parasagittal mass on the right side of the caudal fossa, ventral to the cerebello-pontine angle (Figs. 1-3). The mass had a slight hypointense signal on T1-weighted images and the reticular formation, middle cerebellar peduncle and pons were displaced dorsomedially. On T2-weighted images, the mass was not clearly visualized (Fig. 4). After contrast medium administration, marked homogeneous enhancement was observed. Significant atrophy of the masticator muscles was also seen, especially in the temporalis muscle. There was no sign of muscular infiltration, edema or tissue changes at this level. The lesion was compatible with a schwannoma of the right trigeminal nerve. 
Any surgical attempt was rejected by the owners. Prednisolonell $(1 \mathrm{mg} / \mathrm{kg} / \mathrm{day}$, $\mathrm{PO})$ resulted in disappearance of pruritis, although the muscular atrophy remained unchanged. After 2 months of therapy, progressive weight loss developed. The animal was euthanized 3 months after initial presentation. Necropsy was not performed.

\section{Discussion}

In one report, peripheral nerve sheath tumors represented $26.6 \%$ of nervous system tumors, but primary cranial nerve tumors are seldom found. ${ }^{2}$ In a report of about 60 peripheral nerve sheath tumors, 39 involved spinal nerves, 17 involved peripheral nerves and only 4 involved cranial nerves. ${ }^{3}$ Histologically, tumors of the nerves and nerve sheaths are classified as schwannomas, neurofibromas or neurofibrosarcomas. ${ }^{4}$ Schwannomas and neurofibromas have been described in dogs and cattle while neurofibrosarcomas are found mainly in dogs and rarely in horses and cats. ${ }^{4,5}$ Schwannomas are usually found in the intracranial portion of the cranial nerves and are sometimes the cause of compression of neighboring structures. Cranial nerves most frequently affected in the dog are, in decreasing order, the vestibulocochlear, facial and trigeminal nerves. ${ }^{6}$ 
The most consistent abnormality present with unilateral trigeminal nerve disease is temporalis and masseter muscle atrophy. Polyneuropathies can also be responsible for atrophy of these muscles but almost never in an isolated pattern. Diagnosis may be confirmed with electrophysiologic tests. Polymyositis and hyperadrenocorticism may result in masseter muscular atrophy, but clinical signs are most frequently bilaterally symetrical. The clinical presentation of hyperesthesias and dysesthesias in the trigeminal nerve territory, as observed in this dog, has been often described in humans but is rarely observed in animals. ${ }^{7,8}$

Muscle atrophy as well as spontaneous electrical muscle activity can be neurogenic and/or myogenic in origin. Unfortunately, motor nerve fibers to masticatory muscles are not accessible for direct electrophysiologic differentiation. Thus, a biopsy was performed to exclude primary muscle involvement. Immunohistochemical studies were not conducted in this dog. These may have allowed identification of features more typical of slowly progressive muscle denervation, such as fiber type grouping. Provided that the facial nerves are intact, a trigemenofacial reflex can be quantified in dogs. ${ }^{1}$ This permitted us to confirm sensory fibers involvement. Since the latency was more affected than amplitude, involvement of the nerve sheaths was more likely than that of the axons. Such a presentation was compatible with nerve compression. The absence of other signs 
related to brain stem involvement, and the involvement of both sensory and motor trigeminal fibers, allowed localization of the lesion between the brain stem and the trigeminal ganglion i.e. where sensory and motor fibers are both present. ${ }^{9}$

In humans, trigeminal schwannomas are usually hypo- or isointense on T1weighted images and minimally hypointense on T2-weighted images. ${ }^{10}$ Areas of myxoid degeneration appear hyperintense on $\mathrm{T} 2$ studies. ${ }^{10}$ Lesions invariably enhance intensely with gadolinium. ${ }^{10}$ The lesion described herein had similar characteristics.

A large experience has been gained from surgical treatment of nerve sheath tumors involving nerve roots of the spinal cord cervical enlargement. Recurence of these tumors is the rule and complete cure, the exception. ${ }^{11}$ The common trunk of the trigeminal nerve is the more rostral structure in the caudal fossa and it is located ventromedial to the petrous crest and associated tentorium cerebelli. ${ }^{9} \mathrm{~A}$ transoccipital approach extended through the tentorium cerebelli and squamosal bone might give access to this nerve trunk but would need potentially hazardous venous sinuses pluging and brain structures retraction. 


\section{$\underline{\text { References }}$}

1. Whalen L.R. Electrophysiologic studies of the facial reflexes of the dog. Am J Vet Res 1985;46:229-234.

2. LeCouteur RA. Tumors of the nervous system. In:Withrow SJ, MacEwen EG. Clinical veterinary oncology. Philadelphia: JB Lippincott, 1989; 325.

3. McGrath JT. Morphology and classification of brain tumors in domestic animals. Proceedings of the conference of brain tumors in man and animals, Research Triangle Park, NC, September 5-6, 1984.

4. Bulletin of the world health organisation. International classification of tumours of domestic animals; Part 2. Geneve 1974;50:53-69.

5. Simon J, Brewer RL. Multiple neurofibromatosis in a cow and calf. J Am Vet Med Assoc 1963;142:1102-1104.

6. de Lahunta A. The nervous system. In: Ettinger SJ. Textbook of veterinary internal medicine, 3rd edition. Philadelphia: WB Saunders, 1993;716-717.

7. Chrisman CL. Signs related to other autonomic and somatic cranial nerve dysfunction. In: Chrisman CL. Problems in small animal neurology, 2nd edition. Philadelphia: Lea \& Febiger, 1991;235-266.

8. Shell L. Cranial nerve disorders in dogs and cats. Comp Cont Educ 1982;4:458467. 
9. Evans HE, Kitchell RL. Cranial nerve and cutaneous innervation of the head. In: Evans HE. Miller's anatomy of the dog, 3rd edition. Philadelphia: W.B.Saunders, 1993.

10. Yuh WT, Wright DC, Barloon TJ et al. MR Imaging of primary tumors of trigeminal nerve and Meckel's cave. AJNR 1988;9:665-670.

11. Bailey C.S. Long term survival after surgical excision of a schwannoma of the sixth cervical spinal nerve in a dog. J Am Vet Med Assoc 1990;196: 754-756.

*. Domitor, Smith Kline, Louvain la Neuve, Belgium

$\dagger$. Penthotal, Abbott, Ottignies, Belgium

†. Magnetom, Siemens, Erlagen, Germany

$\S$. Dotarem, Guerbet, Aulnay-sous-bois, France

॥. Medrol, Upjohn, Puurs, Belgium 
$\underline{\text { Caption of figures }}$

Figure 1: A. T1-weighted transverse image. There is an isointense, right parapontine, extra-axial mass which displaces the pons contralaterally (arrowhead). Note the severe ipsilateral masticator muscle atrophy. B. Intense, homogeneous enhancement after intravenous gadolinium administration (arrowhead). 
Figure 2: A. T1-weighted dorsal image. The same mass and displacement of the pons (arrowhead) are visible. B. Post-contrast image at the same level. There is intense enhancement (arrowhead).

Figure 3: T1-weighted sagittal image after gadolinium enhancement. Note the dorso-caudal displacement of the pons by a hyperintense mass (arrowheads).

Figure 4: T2-weighted dorsal view. On this sequence, the mass is isointense to the adjacent pons (arrowhead). Note its extra-axial location, outlined by the hyperintense cerebrospinal fluid. 